\title{
From SARS-CoV-2 infection to COVID-19 morbidity: an in silico projection of virion flow rates to the lower airway via nasopharyngeal fluid boluses*
}

\author{
Saikat Basu', Mohammad Mehedi Hasan Akash', Natasha S. Hochberg²,3, \\ Brent A. Senior ${ }^{4}$ Diane Joseph-McCarthy ${ }^{5,6}$, Arijit Chakravarty ${ }^{7}$ \\ ' Department of Mechanical Engineering, South Dakota State University, SD, United States \\ 2 Department of Medicine, Section of Infectious Diseases, Boston University, MA, United States \\ ${ }^{3}$ Department of Epidemiology, School of Public Health, Boston University, MA, United States \\ ${ }^{4}$ Department of Otolaryngology - Head and Neck Surgery, University of North Carolina at Chapel Hill, NC, United States \\ ${ }^{5}$ Department of Biomedical Engineering, Boston University, MA, United States \\ ${ }^{6}$ Bioengineering Technology and Entrepreneurship Center, Boston University, MA, United States \\ Fractal Therapeutics, MA, United States
}

Rhinology Online, Vol 5: 10 - 18, 2022 http://doi.org/10.4193/RHINOL/21.053

*Received for publication:

October 25, 2021

Accepted: January 7, 2022

Published: January 26, 2022

\begin{abstract}
Background: While the nasopharynx is initially the dominant upper airway infection site for SARS-CoV-2, the physiologic mechanism launching the infection at the lower airway is still not well-understood. Based on the rapidity of infection progression to the lungs, it has been hypothesized that the nasopharynx may be acting as the primary seeding zone for subsequent contamination of the lower airway via aspiration of virus-laden boluses of nasopharyngeal fluids.
\end{abstract}

Methodology: To examine the plausibility of the aspiration-driven mechanism, we have computationally tracked the inhalation process in three anatomic airway reconstructions and have quantified the nasopharyngeal liquid volume transmitted to the lower airspace during each aspiration.

Results: Extending the numerical trends on aspiration volume to earlier records on aspiration frequencies indicates a total aspirated nasopharyngeal liquid volume of $0.3-0.76 \mathrm{ml} /$ day. Subsequently, for mean sputum viral load, our modeling projects that the number of virions reaching the lower airway will range over $2.1 \times 10^{6}-5.3 \times 10^{6} /$ day; for peak viral load, the corresponding number hovers between $7.1 \times 10^{8}-1.8 \times 10^{9}$.

Conclusions: The virion transmission findings fill in a key piece of the mechanistic puzzle on the systemic progression of SARSCoV-2, and subjectively point to health conditions like dysphagia, with proclivity to increased aspiration, as some of the potential underlying risk factors for aggressive lung infections.

Key words: SARS-CoV-2, nasopharyngeal bolus, nose diseases, respiratory system, public health

\section{Introduction}

Severe acute respiratory syndrome coronavirus 2 (SARS-CoV-2), the causative agent for coronavirus disease 2019 (COVID-19), has been linked ${ }^{(1,2)}$ to a remarkable pattern of relatively high infectivity in ciliated epithelial cells along the nasal passage lining in the upper airway, moderate infectivity in cells lining the throat and bronchia, and relatively low infectivity in lung cells. Such viral trends are governed by the abundance of angiotensin-con- verting enzyme 2 (ACE2), a single-pass type I membrane protein that is exploited by viral spike protein binding as a gateway for cellular entry. ACE2 is abundant on ciliated epithelial cells, but is highly expressed in only a smaller subset of the alveolar cells in the lower airway ${ }^{(3)}$. These findings ${ }^{(1,2)}$ are nonetheless for in vitro samples; virus-laden droplets deposited along the anterior nasal airway might not be so effective at launching an infection despite the presence of ciliated cells, as the thicker mucus layer 
Table 1. List of abbreviations (in order of appearance).

\begin{tabular}{ll} 
Acronym & \multicolumn{1}{c}{ Full Name } \\
\hline SARS-CoV-2 & Severe Acute Respiratory Syndrome Coronavirus 2 \\
COVID-19 & Coronavirus Disease 2019 \\
CT & Computed Tomography \\
FESS & Functional Endoscopic Sinus Surgery \\
L/min & Liters per minute \\
DICOM & Digital Imaging and Communications in Medicine \\
STL & Stereolithography \\
CFD & Computational Fluid Dynamics \\
LES & Large Eddy Simulation \\
RNA & Ribonucleic Acid \\
PCR & Polymerase Chain Reaction \\
RT & Reverse Transcription \\
GERD & Gastroesophageal Reflux Disease \\
OSA & Obstructive Sleep Apnea \\
OR & Odds Ratio \\
CI & Confidence Interval \\
CPAP & Continuous Positive Airway Pressure \\
& \\
\hline
\end{tabular}

there provides some level of protection against viral invasion and infection ${ }^{(4)}$. Hence, the nasopharynx, which is the region in the upper airway posterior to the septum and comprising the superior portion of the pharynx, has been postulated to be a dominant initial infection site for SARS-CoV-2 $2^{(1,5)}$. The efficacy of nasopharyngeal swabs over oropharyngeal swabs for accurate detection $^{(6)}$ of COVID-positive cases supports the hypothesis. Based on the brisk pace at which lower airway infections ensue following the emergence of initial symptoms, it has been conjectured ${ }^{(1)}$ that the nasopharynx (marked in Figure 1, see Panels (a)-(i)) acts as the seeding zone for subsequent spread of the disease to the lungs via lower airway aspiration of virus-laden boluses of nasopharyngeal fluids.

While the above hypothesized mechanism is superficially plausible, the key unanswered question is whether the rate of virion flow from the initial site of infection to the lower airway could be sufficiently high to account for a rapid onset of secondary infections at the lungs. In this report, we have combined earlier data on aspiration trend $s^{(7,8)}$ with virological assessments of sputum in hospitalized COVID ${ }^{(9)}$ patients and our computational findings on the physical flow variables in anatomically realistic airway domains - to quantify bolus-borne virion transmission rates from the nasopharynx into the lower airway.

Preliminary results from this study have been presented at the International Society for Aerosols in Medicine (ISAM) Con- gress ${ }^{(10,11)}$, held in May 2021. This work builds on an established line of computational tracking studies of upper airway respiratory transport ${ }^{(12-24)}$.

\section{Materials and methods}

Frequency and quantification of pharyngeal aspiration Aspiration (i.e., accidental suction of fluid and cells into the lungs) of upper airway secretions acts as a major carrier of pathogens to the lower airway, and the phenomenon, fortunately enough, has been studied in great detail over the last few decades. As reported in the late-1990s ${ }^{(7)}$, aspirated pharyngeal liquid volume during sleep ranges from $0.011 \mathrm{ml}$ to $0.129 \mathrm{ml}$, measured through tracking mildly-radioactive tracers after the subjects wake up. Further inspection of the published data indicates that the maximum data-point in the reported range is a statistical outlier. Including the maximum-reported volume in the analysis, the mean aspirated volume comes out to be $0.0345 \mathrm{ml}$ and the median is $0.0215 \mathrm{ml}$. Excluding the outlier, the mean volume revises to $0.021 \mathrm{ml}$ and the median volume adjusts to $0.020 \mathrm{ml}$. That study ${ }^{(7)}$ was based on a total of 10 normal subjects.

While evaluating swallowing mechanisms, it has been further reported $^{(8)}$ that for 5 -ml bolus volumes, aspirations happen during $13 \%$ of swallows; and for $10-\mathrm{ml}$ bolus volumes, aspirations happen during $11 \%$ of swallows. Boluses smaller than $10 \mathrm{ml}$ are associated with silent aspiration ${ }^{(25)}$ and presumably are the major sources of pathogen-carriers to the deep lungs, and with averaging the reported data for $5-\mathrm{ml}$ and $10-\mathrm{ml}$ volumes, $12 \%$ of the swallowing actions should result in aspiration.

Finally, earlier findings ${ }^{(26)}$ suggest that a typical person swallows 500 - 700 times during a day and 24 times during sleep (assuming a standard eight-hour sleep cycle). These numbers thus indicate that a subject will aspirate approximately $12 \%$ of 500 700 times, i.e. $60-84$ times during the day, and $12 \%$ of 24 times, i.e., approximately 3 times during sleep.

Development of anatomically realistic computational fluid mechanics models

Allometric relationships ${ }^{(27)}$ show that the minute inhalation is approximately $14.5-20.0 \mathrm{~L} / \mathrm{min}$ for a $65-\mathrm{kg}$ adult male and 8.8 - 22.4 L/min for a 65-kg adult female, both for gentle steady breathing. For simplicity, as a stand-in for gentle inhalation ${ }^{(28-32)}$, this study simulates an airflow of $15 \mathrm{~L} / \mathrm{min}$; the process can be modeled using viscous-laminar steady state flow physics schemes. We additionally noted that subjects with proclivity to apnea are also prone to higher levels of aspiration ${ }^{(33)}$ and for them, the inhaled air often disrupts into high-speed turbulent regimes. To account for such flow conditions, we have simulated a higher breathing rate of $55 \mathrm{~L} / \mathrm{min}$. 


\section{Anatomic airway reconstructions}

The in silico anatomic geometries were reconstructed from medical-grade computed tomography (CT) scans sourced from existing de-identified imaging data of the upper respiratory tract. Use of the archived records was approved with exempt status by the Institutional Review Board of the University of North Carolina (UNC) at Chapel Hill, with the informed consent requirement waived for retrospective computational use. The test subjects include a 61-year-old female (named hereafter as Subject 1), a 37-year-old female (named as Subject 2), and a 24-year-old female (named as Subject 3). Subjects 1 and 2 presented clinically normal CT scans, while Subject 3 had a post-FESS (Functional Endoscopic Sinus Surgery) airway with a diagnosed pre-procedural complication of Chronic Rhinosinusitis.

As for imaging resolution, the CT slices were collected at coronal depth increments of $0.4 \mathrm{~mm}$. The anatomic airspace was extracted from the scans over a delineation range of -1024 to -300 Hounsfield units, the process was complemented by careful hand-editing of the selected pixels for anatomic accuracy. This phase of digital reconstruction was carried out by processing the DICOM (Digital Imaging and Communications in Medicine) scans of the subjects' airways on the image processing software Mimics Research v18.0 (Materialise, Plymouth, MI, USA). Subsequently, the reconstructed geometries were exported as STL (stereolithography) files to ICEM-CFD 2019 R3 (ANSYS Inc., Canonsburg, PA, USA) and therein were spatially meshed into minute volume elements. As per established mesh refinement protocols $^{(34-36)}$ and other more recent reports ${ }^{(37,38)}$, each computational grid in this study contains more than 4 million unstructured, graded tetrahedral elements (namely 4.54 million in Subject 1, 4.89 million in Subject 2, and 4.05 million in Subject 3).

\section{Simulating inhalation}

Inhaled transport was studied through computational fluid dynamics (CFD) in the three meshed geometries (Subjects $1-3$ ), for two different inhaled airflow rates, 15 and $55 \mathrm{~L} / \mathrm{min}$. To track the $15 \mathrm{~L} / \mathrm{min}$ flow, viscous-laminar steady state simulations of the inhalation process were carried out using a segregated solver on ANSYS Fluent 2019 R3, with SIMPLEC pressure-velocity coupling and second-order upwind spatial discretization. We monitored the solution convergence by minimizing the mass continuity and velocity component residuals, and by stabilizing the mass flow rate and static pressure at the airflow outlets. For the pressuredriven flow solutions, typical run-time for 5000 iterations was 2-3 hours through 4 processor-based parallel computations executed at $3.1 \mathrm{GHz}$ speed on Xeon nodes. The higher flow rate of $55 \mathrm{~L} / \mathrm{min}$ results in turbulence $\mathrm{e}^{(22,23,39-41)}$, which we have tracked via Large Eddy Simulations (LES), with Kinetic Energy Transport Model as the sub-grid scale model ${ }^{(42)}$. Each LES computation required a run-time of 1-2 days, for a simulated flow interval of 0.25 second, with the time-step at 0.0001 second. To account for the elevated temperatures inside the airspace, the simulations implemented an air density of $1.204 \mathrm{~kg} / \mathrm{m}^{3}$ and $1.825 \times 10^{-5}$ $\mathrm{kg} / \mathrm{m} . \mathrm{s}$ was used as the dynamic viscosity of air.

The following boundary conditions were enforced in the flow simulations: zero velocity (no slip) at the internal airway walls, i.e., at the tissues and cartilages enclosing the airway; zero pressure at nostril openings, which acted as the pressure-inlet zones; and negative pressure at the airflow outlet at the base of the nasopharynx, which acted as the pressure-outlet zone. Review Figure 1 for the relative locations of the anatomic regions.

\section{Theoretical estimation of aspirated nasopharyngeal liquid volume}

For an instantaneous pathogenic event such as pharyngeal aspiration, the transport of the nasopharyngeal liquids downstream to the lower airway can be physically described through a reduced-order mathematical model of steady unidirectional flow, wherein the liquid bolus originates at the nasopharyngeal tissue surfaces through shearing action and subsequently escapes to the lower airway under the pressure gradient imposed by the respiratory mechanism.

With the assumption of axial symmetry in the airway conduit and no slip boundary condition at the walls, integrating the Navier-Stokes equation for momentum conservation results ${ }^{(43-46)}$ in: $\mathrm{Q}=-\left[\pi \mathrm{R}^{4} /(8 \mu)\right](d p / d z)$. Here, $\mathrm{Q}$ is the instantaneous aspirated volume, $\mathrm{R}$ is the hydraulic radius (cross-section of conduit divided by the perimeter at the nasopharyngeal base), $\mu$ is the sputum viscosity (quantified at 4.59 poise $=0.459 \mathrm{~Pa}^{\mathrm{sec}} \mathrm{s}^{(47)}$ for mucopurulent medium), and $d p / d z$ is the spatial rate of pressure gradient in the streamwise direction. To adapt the formulation to the present problem, we have post-processed the simulations to extract the averaged wall pressure at the nasopharynx (pn). We have also extracted the averaged pressure at the outlet (po), located $0.02 \mathrm{~m}$ (streamwise $\Delta Z$, to ensure full flow development in the simulations) below the nasopharyngeal base. With $\triangle P=$ $p o-p n$, the gradient rate $d p / d z$ can therefore be approximated to simply $\Delta P / \Delta Z$.

\section{Data on viral loading}

SARS-CoV-2 is a single-stranded RNA virus (i.e., comprising single-stranded genomes made of ribonucleic acid), and the virological assessments ${ }^{(9)}$ performed through PCR (polymerase chain reaction) with reverse transcription (RT-PCR) on the sputum (collected via nasopharyngeal swabs) of hospitalized COVID-19 patients indicate a mean viral load of $v_{\text {avg }}=7 \times 10^{6} \mathrm{RNA}$ copies $/ \mathrm{ml}$ of oral fluid. The peak load was $v_{\text {peak }}=2.35 \times 10^{9}$ copies $/ \mathrm{ml}$. 


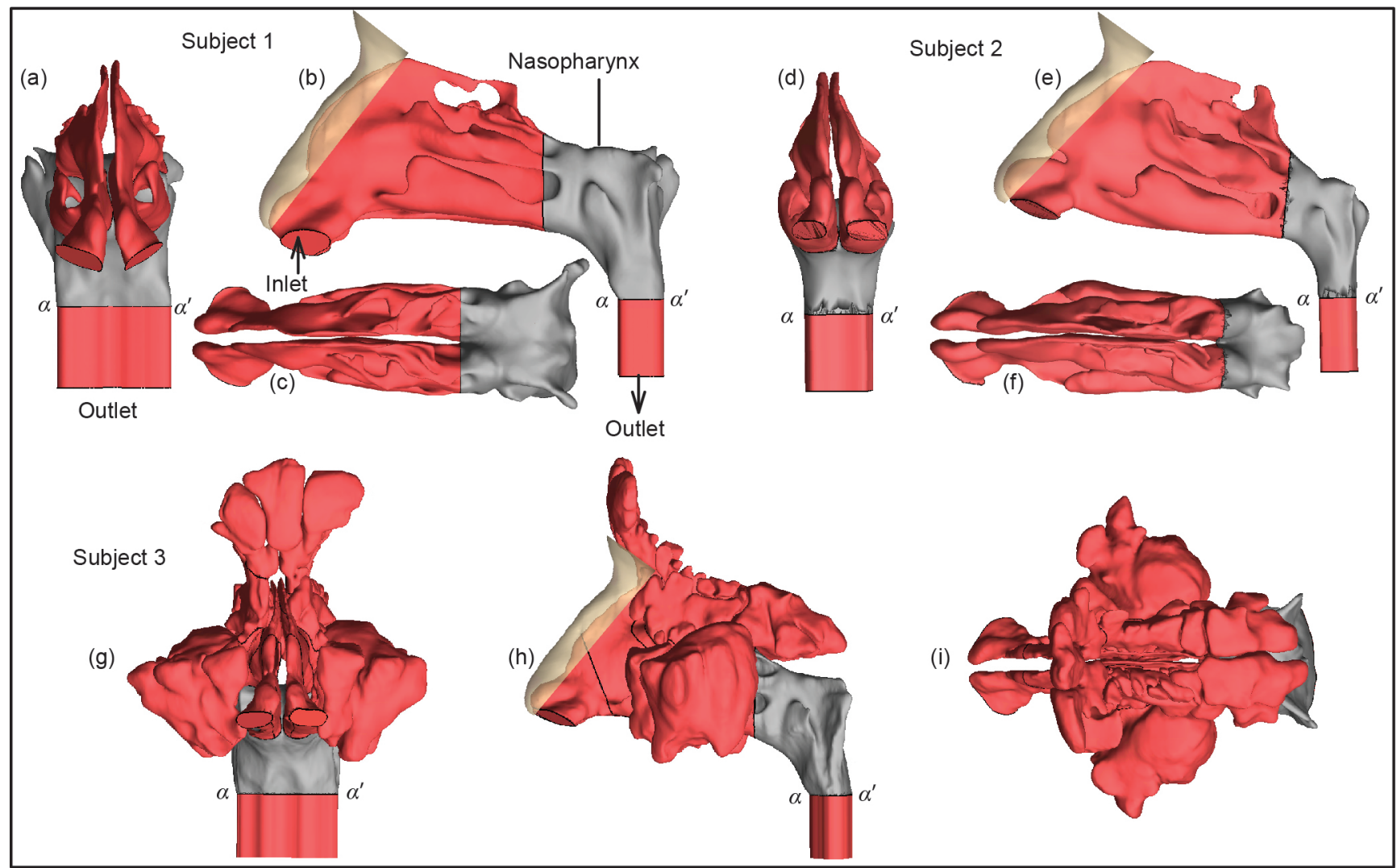

Figure 1. Panels (a), (b), and (c) respectively show the coronal, sagittal, and axial views of the computed tomography (CT) based upper airway reconstructions for Subject 1. Panels (d), (e), and (f) show the corresponding views for Subject 2. Panels (g), (h), and (i) depict the corresponding views for Subject 3 (comprising a full sinonasal domain). The nasopharynx has been marked in grey in each subject. Additionally, a-a' shows the location of the wetted perimeter used while estimating the hydraulic radius (refer to the Results section).

\section{Results}

Computational prediction of nasopharyngeal bolus volume With inhalation simulated at $15 \mathrm{~L} / \mathrm{min}$ : for Subject $1, \Delta P$ was -0.82 Pa; for Subject 2, $\triangle P$ was $-2.33 \mathrm{~Pa}$ (for contour maps, see Figure 2, Panels (a) and (b)), and for Subject 3, $\triangle P$ was $-0.46 \mathrm{~Pa}$. The hydraulic radius in Subject 1 was $3.43 \mathrm{~mm}$, i.e. $R=0.00343$ $\mathrm{m}$; the hydraulic radius in Subject 2 was $3.06 \mathrm{~mm}$, i.e. $R=0.00306$ $\mathrm{m}$; and the hydraulic radius in Subject 3 was $4.08 \mathrm{~mm}$, i.e. $\mathrm{R}=$ $0.00408 \mathrm{~m}$. Consequently using the mathematical formulation of $Q$ from the Methods in Subject 1: the liquid bolus volume transmission rate in each aspiration is $\pi \times R 4 \times|\Delta P| /(8 \times 0.459 \times 0.02)$ $=4.86 \times 10^{-9} \mathrm{~m}^{3}=0.00486 \mathrm{ml}$. Similarly, in Subject 2: the liquid bolus volume ingested during each aspiration is $0.00874 \mathrm{ml}$; in Subject 3: the liquid bolus volume ingested during each aspiration is $0.00545 \mathrm{ml}$.

\section{Estimation of aspiration frequency and validation of the computational predictions}

Using the earlier observational data in the first paragraph under Methods, in conjunction with the range of the computed volumes above, the aspiration frequency during each sleep cycle is on the order of $(0.021 \mathrm{ml}) /(0.00874 \mathrm{ml})$ and $(0.021 \mathrm{ml}) /(0.00486$ $\mathrm{ml}$ ), or approximately $2-4$ times. Calculations based on direct experimental observations provide an estimate of 3 aspirations during a sleep cycle (derived in the second and third paragraphs under Methods using previous reports $\left.{ }^{(8,26)}\right)$. The computational framework thus offers strong agreement with the direct experimental observations, providing a measure of validation for the underlying basic fluid dynamics framework.

\section{Estimation of virion flow to the lower airway}

Total number of aspirations of liquid boluses into the lower airway approximately ranges between $63-87$ times in a day (see Methods, note that the estimate includes the nocturnal aspirations during the subdued sleep phase). Based on the data from the current subjects in the numerical simulations (see Results), the total volume of aspirated liquid in a day is thus between $0.00486 \times 63 \mathrm{ml}$ and $0.00874 \times 87 \mathrm{ml}$, or between 0.3 $-0.76 \mathrm{ml}$. With the virological data from the last section in the Methods, the number of virions penetrating the lower airway per day, while suspended in the nasopharyngeal fluid boluses, is therefore between $0.3 \times v_{\text {avg }}=2.1 \times 10^{6}$ and $0.76 \times v_{\text {avg }}=5.3 \times 10^{6}$, when considering the mean viral load. For peak viral load $v_{\text {peak }}$ in the sputum, the corresponding number of penetrating virions 


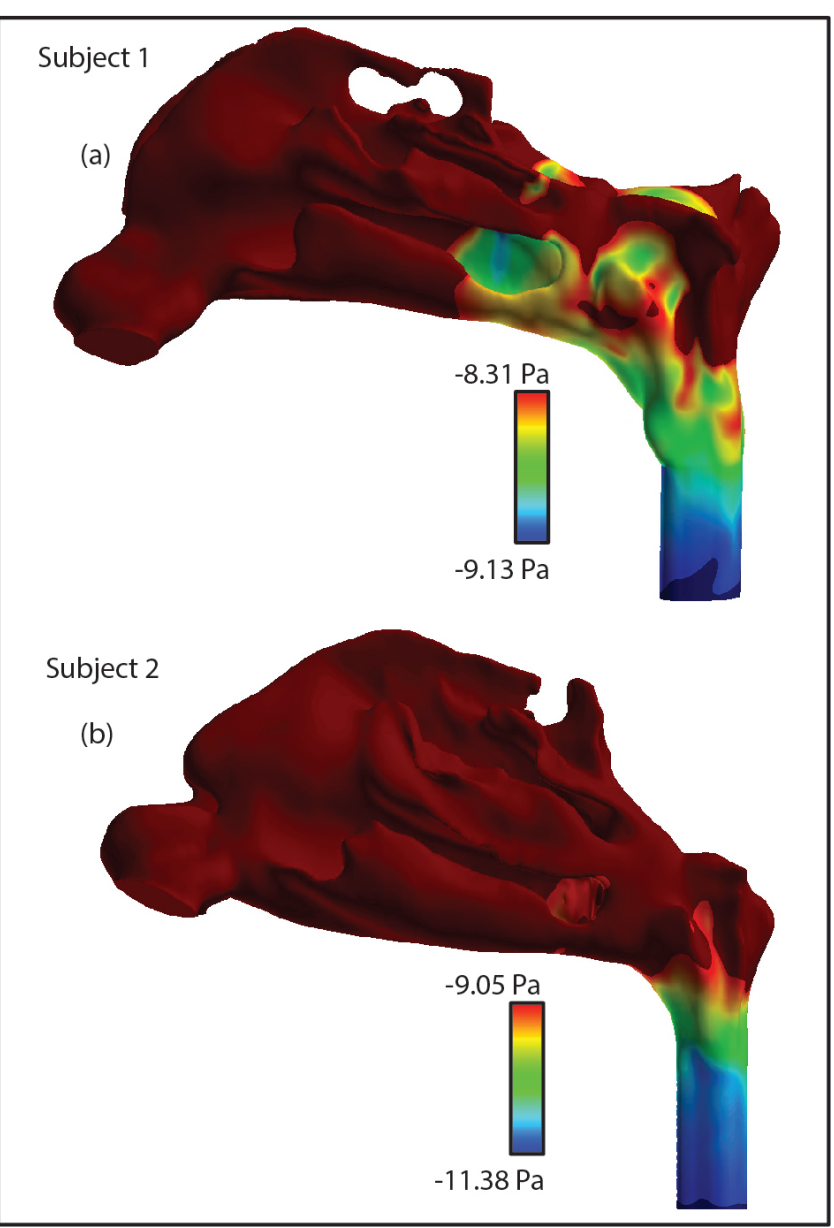

Figure 2. Panels (a) and (b) respectively depict the wall pressure maps in Subject 1 and Subject 2, for simulated inhalation of $15 \mathrm{~L} / \mathrm{min}$. Note that the pressure color-maps set the limiting contour colors at the respective pn (averaged wall pressure at nasopharynx) and po (averaged pressure at outlet) values that are extracted from the simulated data in each case. The data post-processing is done on FieldView v18.0, under software license provided through the University Partners Program.

ranges over $7.1 \times 10^{8}-1.8 \times 10^{9}$ per day. Such virion transmission numbers are indeed a high estimate when compared to the infectious dose for SARS-CoV-2, which has been detected to be of the order $\mathrm{O}\left(10^{2}\right)^{(48-50)}$, or more precisely ${ }^{(5)} \sim 300$, a number supported by an earlier study ${ }^{(51)}$ on coronaviruses. The infectious dose is a fundamental virological measure quantifying the minimum number of virions that can go on to launch an infection in a subject.

The higher inhalation rate, i.e. $55 \mathrm{~L} / \mathrm{min}$, results in: $\Delta P=-8.41$ $P a$ in Subject 1; $\Delta P=-25.37 \mathrm{~Pa}$ in Subject 2; and $\Delta P=-4.73 \mathrm{~Pa}$ in Subject 3 . Therefore, the growth in $|\Delta P|$ stays consistently 10 -fold (when compared to the corresponding pressure differentials at $15 \mathrm{~L} / \mathrm{min}$, see earlier in the Results); the growth in virion transmission would also consequently be 10 -fold.

\section{Discussion}

We have used a novel in silico approach to estimate virion flow rates via aspiration of virus-laden boluses of nasopharyngeal fluids from the initial dominant infection site in the upper airway (i.e., the nasopharynx) to the lungs. Our findings suggest that aspiration of mucosal fluid represents a simple yet viable mechanism for the rapid spread of SARS-CoV-2 infections from the upper respiratory tract (in particular the nasopharynx) to the lungs.

The numerical projections have been validated at each step along the way by comparing them to experimental observations. First, the computational prediction of the aspirated liquid volume in each occurrence is validated by comparing the number of projected aspirations (approximately $2-4$ ) during an eight-hour sleep cycle with prior observational findings of 3 aspirations in human subjects. Next, the aspiration volumes projected by us have been linked with earlier findings on aspiration frequency for the entire day, to obtain a total aspirated nasopharyngeal liquid volume of $0.3-0.76 \mathrm{ml}$ each day. Finally, we can compare our estimates for viral load delivered to the lower airway to the estimates for minimum infectious dose for SARS-CoV-2. Using virological assessment data in the oral fluids collected from hospitalized COVID-19 patients ${ }^{(9)}$, we project that the number of virions penetrating the lower airway daily would range over $2.1 \times 10^{6}-5.3 \times 10^{6}$, for mean viral load. When the viral load peaks, our projections suggest that the number of penetrating virions increases to $7.1 \times 10^{8}-1.8 \times 10^{9}$ per day. The minimum infectious dose for SARS-CoV-2 transmission from one human host to another is on the order of $O\left(10^{2}\right)$ virions ${ }^{(50)}$ (as estimated by deep sequencing studies ${ }^{(52)}$, and also through our earlier computational findings ${ }^{(5)}$ that merged fluid dynamics-based tracking of inhaled transport with the related virologic assessments). Thus, it stands to reason that a dose $10^{4}-10^{7}$ times higher (considering gentle inhalation in the host) will suffice to seed a second infection site within the same host, particularly given the relatively high levels of ACE2 expression in a subset of alveolar cells ${ }^{(3)}$.

Role of boundary layer separation in generation of aspirates We believe that the results of our CFD modeling also point to a role for the nasopharynx specifically in seeding infections to the lungs. The wall pressure differences between the anterior nasal sites (e.g., the turbinates, the olfactory cleft) and the airway outlet in the test subjects are found to be of approximately the same order of magnitude as that between the nasopharynx and the outlet (which was -1.2 Pa, averaged over the test subjects). For example, in Subject 3, the pressure difference was -1.15 Pa between the left middle turbinate and the outlet, $-2.59 \mathrm{~Pa}$ between the outlet and the left olfactory roof, $-2.90 \mathrm{~Pa}$ between the outlet and the right middle turbinate, and $-2.85 \mathrm{~Pa}$ between the outlet and the right olfactory roof. However, quite critically, 
the nasopharyngeal bolus generation (and consequently, the aspiration) is predominantly triggered by the mechanistic process of shear-induced ${ }^{(53,54)}$ flow separation. Boundary layer separation (or flow separation) entails the detachment of walladhering fluids owing to adverse pressure gradient imposed on the boundary layers by the outer potential flow ${ }^{(55)}$. This happens when the outer flow abruptly alters its mean direction, e.g., at the $\sim 90^{\circ}$ bend of the nasopharynx. Thus, the role of the nasopharynx in generation of virus-laden boluses of mucosal fluid is presumably due both to the pressure differential (a necessary but not sufficient condition) and the unique geometry of the region.

\section{Clinical ramifications}

The findings suggest a simple aspiration-based physiological mechanism for COVID-19 etiology following initial SARS-CoV-2 infection in the nasopharynx. Such a mechanistic link may be valuable in identifying risk factors that predispose patients to progress to acute COVID-19 morbidity following SARS-CoV-2 infection. For example, a prediction that emerges readily from this proposed mechanism is that individuals with dysphagia may be at increased risk of developing COVID-19 following SARS-CoV-2 onset and may have more negative outcomes with the disease. In this context, the reader should note that while "dysphagia" is a broad clinical condition, we are specifically using the term here to refer to situations where aspiration or microaspiration may occur with, for instance, difficulty in swallow initiation.

One condition associated with dysphagia is obstructive sleep apnea (OSA) ${ }^{(56-58)}$, with increased nocturnal aspiration ${ }^{(33)}$ and risk of aspiration-induced pneumonia ${ }^{(59)}$. Based on the mechanism proposed by us for viral spread to the lungs, individuals with OSA would thus be expected to be at a higher risk for COVID-19 (or, for that matter, any progressive respiratory viral disease). In fact, this has been reported by several different investigators $^{(60-64)}$. One study, for example, reported an association of OSA with increased risk for hospitalization (OR 1.65; $95 \% \mathrm{CI}$ $(1.36,2.02))$ and respiratory failure (OR 1.98; 95\% Cl $(1.65,2.37))$ owing to COVID-19, after adjusting for diabetes, hypertension, and body mass index ${ }^{(65)}$. As a practical matter, our work suggests that individuals with OSA should not suspend the use of their CPAP (continuous positive airway pressure) devices upon testing positive for SARS-CoV-2, as has been suggested by some practitioners ${ }^{(66)}$.

As the prevalence of dysphagia also increases with other factors such as increased age ${ }^{(67)}$, cancer treatment ${ }^{(68)}$, and Parkinsonism ${ }^{(69)}$, the physiological mechanism proposed here may also account for some of the documented increased risk of adverse outcomes $^{(70-73)}$ in these groups. Any sedation outside of the hospital (e.g., from alcohol / substance use) or within the hospital (from sedating medications) might also trigger increased aspiration. Further study is required to understand the importance of dysphagia in general as a predictive factor for adverse outcomes with COVID-19.

Our findings also point to the potential risk posed by vaccine breakthrough cases. We now know that vaccines against SARSCoV-2 do not provide sterilizing immunity - recent real-world data points to a reduction in risk of infection for vaccinated individuals that ranges from $20-50 \%$ of that of unvaccinated individuals ${ }^{(74)}$. Such a scenario is of particular concern for the current wave of vaccines that target the spike protein; work by $u s^{(75)}$ and others ${ }^{(76-79)}$ suggests that the spike protein has a relatively high mutational tolerance and can readily generate immuneevading mutations now that vaccinal and natural immunity are widespread within the population.

Thus, given that vaccinal prophylaxis does not provide sterilizing immunity, vaccinated individuals infected with SARS-CoV-2 in the nasopharynx are being subjected to a steady stream of virions testing their immune system with potential immuneevading mutations. Some proportion of patients who experience breakthrough infections are therefore likely doing so as a result of intrahost evolutionarily-mediated resistance, which has been reported by $\mathrm{us}^{(80)}$ and others to be a significant risk for SARS-CoV-2 infections. These infections can go on to seed transmission chains of vaccine-resistant SARS-CoV- 2 within the population. This suggests that breakthrough infections, even asymptomatic ones, are likely providing an engine for the evolution of vaccine resistance. Consistent with this hypothesis, a comprehensive analysis of 1.5 million SARS-CoV-2 genomes (with whole-genome sequencing of vaccine breakthrough cases) showed evolutionarily mediated immune evasion to be a common feature of vaccine breakthrough ${ }^{(52,74,76)}$. Critically, our work thus suggests that the current public health strategy of using vaccines to limit severe disease while ignoring breakthrough infections - particularly asymptomatic ones ${ }^{(81)}$ - may underestimate the systemic threat posed by nasopharyngeal SARS-CoV-2 infections.

\section{Study limitations}

While the general fluid mechanics framework of our study is extensible to other respiratory pathogens, our results should still be interpreted as being preliminary, given that the numerical findings are based on simulated data from only three test subjects. We, however, note that there is a good agreement between the numerical predictions of aspiration frequency with earlier observational findings, which lends to the validity of the underlying computational and mathematical framework. The size of the projected viral dose also speaks to the robustness of the conclusion - it is likely that virion flow from the nasopha- 
rynx to the lungs occurs in large excess of the minimum dose required to seed a lung infection for many individuals, thereby precipitating a brisk aggravation of the disease symptoms.

Note that, from a clinical standpoint, we have not considered the contribution of mucociliary activity, propelling a bolus toward the oropharynx from the nasopharynx. However, while that would theoretically increase the bolus volume and the potential frequency of aspiration, it would also provide a protective mechanism after aspiration, as the bolus could be propelled up through a reflux mechanism at the trachea.

Finally, the aspirated pharyngeal liquid volumes could be significantly variable depending on other disease processes, including gastroesophageal reflux disease (GERD), which is very common in the United States ${ }^{(82,83)}$.

\section{Conclusion}

Although the nasopharynx stands out as the dominant initial infection site for SARS-CoV-2, the physiological mechanism launching the lower airway infection is still not well-understood. Given the rapidity of systemic infection progress, it is hypothesized that the nasopharynx acts as the seeding zone for subsequent contamination of the lower airway via aspiration of virus-laden boluses of nasopharyngeal fluids. We have examined the plausibility of this transport mechanism through state-ofthe-art CFD models of steady and forced breathing in three tomographic airway reconstructions, thereby quantifying the nasopharyngeal liquid volume transmitted to the lower airspace in each aspiration. Our physics-based model predicts 2-4 aspirations during an eight-hour sleep cycle, consistent with prior experimental data. Extending the numerical trends on aspiration volume to earlier records on aspiration frequency indicates a total aspirated nasopharyngeal liquid volume of $0.3-0.76 \mathrm{ml} /$ day. Using sputum viral loads for hospitalized COVID-19 patients, we have then estimated the number of virions transmitted daily to the lungs via nasopharyngeal liquid boluses. For peak sputum viral load, the number is $7.1 \times 10^{8}-1.8 \times 10^{9}$ virions/day, well more than the estimated minimum infectious dose for SARS-CoV-2. These findings thus back a plausible mechanism for the brisk progression of SARS-CoV-2 infection of the nasopharynx to the COVID-19 disease within a patient and subjectively point to dysphagia as one of the potential underlying risk factors for adverse outcomes.

\section{Authorship contribution}

SB and AC conceptualized the study and co-drafted the manuscript; SB developed the in silico anatomic geometries and performed the computational simulations; MMHA assisted in revising the manuscript and in running complementary breathing simulations; NSH and BAS provided inputs on the clinical components and the associated commentary; BAS additionally oversaw the recruitment of test subjects; DJM provided inputs on the study design, the data analysis, and critiqued the manuscript.

\section{Acknowledgments}

The researchers accessed computing facilities at both South Dakota State University and UNC Chapel Hill, to derive the reported in silico findings.

\section{Funding}

This work was partially supported by the National Science Foundation (NSF) RAPID Grant \# 2028069 for COVID-19 research, with SB as the Principal Investigator. Any opinions, findings, and conclusions or recommendations expressed here are, however, those of the authors and do not necessarily reflect views of the NSF.

\section{Ethics approval and consent to participate}

No approval or consent needed.

\section{Consent for publication}

Not applicable.

\section{Availability of data and materials}

On request, all data and study protocol can be made available.

\section{Conflict of interest}

The authors declare no competing interests.

\section{References}

1. Hou YJ, Okuda K, Edwards CE, et al. SARSCoV-2 reverse genetics reveals a variable infection gradient in the respiratory tract. Cell. 2020;182(2):429-446. e414.

2. Matheson NJ, Lehner PJ. How does SARS-CoV-2 cause COVID-19? Science. 2020;369(6503):510-511.

3. Ortiz ME, Thurman A, Pezzulo AA, et al. Heterogeneous expression of the SARS Coronavirus-2 receptor ACE2 in the human respiratory tract. EBioMed. 2020;60:102976.
4. Mittal R, Ni R, Seo J-H. The flow physics of COVID-19. J Fluid Mech. 2020;894.

5. Basu S. Computational characterization of inhaled droplet transport to the nasopharynx. Sci Rep. 2021;11(1):1-13.

6. Patel MR, Carroll D, Ussery E, et al. Performance of oropharyngeal swab testing compared with nasopharyngeal swab testing for diagnosis of coronavirus disease 2019-United States, January 2020-February 2020. Clin Infect Dis. 2021;72(3):482-485.
7. Gleeson K, Maxwell SL, Eggli DF. Quantitative aspiration during sleep in normal subjects. Chest. 1997;111(5):1266-1272.

8. Butler SG, Stuart A, Leng X, Rees C, Williamson J, Kritchevsky SB. Factors influencing aspiration during swallowing in healthy older adults. Laryngoscope. 2010;120(11):2147-2152.

9. Wölfel R, Corman VM, Guggemos W, et al. Virological assessment of hospitalized patients with COVID-2019. Nature. 2020;581(7809):465-469. 
10. Basu S, Hochberg N, Senior B, JosephMcCarthy D, Chakravarty A. Computational projection of virion transmission rates to the lower airway from the initial SARSCoV-2 infection at the Nasopharynx. J Aerosol Med Pulmonary Drug Del. 2021:A8A9.

11. Basu S, Hochberg NS, Senior BA, JosephMcCarthy D, Chakravarty A. Computational projection of virion transmission rates to the lower airway from the initial SARSCoV-2 infection at the nasopharynx. Proceedings of the XXIII Congress of the International Society for Aerosols in Medicine (ISAM). 2021.

12. Inthavong K, Ma J, Shang Y, et al. Geometry and airflow dynamics analysis in the nasal cavity during inhalation. Clin Biomech. 2019;66:97-106

13. Zhang $Y$, Shang $Y$, Inthavong $K$, et al. Computational investigation of dust mite allergens in a realistic human nasal cavity Inhal Toxicol. 2019:31(6):224-235.

14. Basu S, Frank-Ito DO, Kimbell JS. On computational fluid dynamics models for sinonasal drug transport: Relevance of nozzle subtraction and nasal vestibular dilation. Int J Numer Method Biomed Eng. 2018:34(4):e2946.

15. Farzal Z, Basu S, Burke A, et al. Comparative study of simulated nebulized and spray particle deposition in chronic rhinosinusitis patients. Int Forum Allergy Rhinol. 2019 Jul; 9(7):746-758

16. Kiaee M, Wachtel H, Noga ML, Martin AR, Finlay WH. Regional deposition of nasal sprays in adults: A wide ranging computational study. Int J Numer Method Biomed Eng. 2018;34(5):e2968.

17. Zhao K, Scherer PW, Hajiloo SA, Dalton P. Effect of anatomy on human nasal air flow and odorant transport patterns: implications for olfaction. Chem Senses. 2004;29(5):365-379.

18. Xi J, Longest PW. Numerical predictions of submicrometer aerosol deposition in the nasal cavity using a novel drift flux approach. Int J Heat Mass Transfer. 2008;51(23-24):5562-5577.

19. Shanley KT, Zamankhan P, Ahmadi G, Hopke PK, Cheng Y-S. Numerical simulations investigating the regional and overall deposition efficiency of the human nasal cavity. Inhal Toxicol. 2008:20(12):1093-1100

20. Kelly J, Prasad A, Wexler A. Detailed flow patterns in the nasal cavity. J Applied Physiol. 2000;89(1):323-337.

21. Basu S, Holbrook LT, Kudlaty K, et al. Numerical evaluation of spray position for improved nasal drug delivery. Sci Reports. 2020;10(1):1-18

22. Tracy LF, Basu S, Shah PV, et al. Impact of endoscopic craniofacial resection on simulated nasal airflow and heat transport. Int Forum Allergy Rhinol. 2019 Aug; 9(8):900909

23. Perkins EL, Basu S, Garcia GJ, Buckmire RA Shah RN, Kimbell JS. Ideal particle sizes for inhaled steroids targeting vocal granulo- mas: preliminary study using computational fluid dynamics. Otolaryngol Head Neck Surg. 2018;158(3):511-519.

24. Julia S, Zanation M, Adam M, et al. Clinical questions and the role CFD can play. Paper presented at: APS Division of Fluid Dynamics Meeting Abstracts2016.

25. Leder SB, Suiter DM, Green BG. Silent aspiration risk is volume-dependent. Dysphagia. 2011;26(3):304-309.

26. Australia SP. Swallowing Awareness Day. speechpathologyaustraliaorgau. 2020

27. Garcia GJ, Schroeter JD, Segal RA, Stanek J, Foureman GL, Kimbell JS. Dosimetry of nasal uptake of water-soluble and reactive gases: a first study of interhuman variability. Inhal Toxicol. 2009;21(7):607-618.

28. Chakraborty A, Jorgensen A, Yuk J, et al. Simulating inhaled transport through bioinspired pathways in mask filters. Paper presented at: APS Division of Fluid Dynamics Meeting Abstracts2020

29. Yuk J, Baskota A, Cooke B, et al. 3D-printing mask filters inspired by animal nasal cavity Paper presented at: APS Division of Fluid Dynamics Meeting Abstracts2020.

30. Chung C-I, Yuk J, Jorgensen A, Basu S, Jung $S$, Chamorro L. Vortex traps to capture particles with reduced pressure loss in respiratory masks. Paper presented at: APS Division of Fluid Dynamics Meeting Abstracts2020.

31. Yuk J, Frohlich K, Connor R, Basu S, Chamorro L, Jung S. Bio-inspired mask filters with breathing resistance control. Bulletin of the American Physical Society. 2021

32. Chakraborty A, Cheng S, Yuk J, et al. Design of new-generation scalable filters with tortuous pathways inspired from animal noses. Bulletin of the American Physical Society. 2021

33. Sato K, Chitose S-i, Sato K, Sato F, Ono T, Umeno $\mathrm{H}$. Sleep-related deglutition and respiratory phase patterns in the aged with obstructive sleep apnea under CPAP therapy. Acta Oto-Laryngol. 2020;140(10):861 868.

34. Frank-Ito DO, Wofford M, Schroeter JD, Kimbell JS. Influence of mesh density on airflow and particle deposition in sinonasal airway modeling. J Aerosol Med Pulm Drug Del. 2016;29(1):46-56.

35. Basu S, Witten N, Kimbell J. Influence of localized mesh refinement on numerical simulations of post-surgical sinonasal airflow. J Aerosol Med Pulmonary Drug Del. 2017 Jun; 30(3):14-14

36. Kimbell JS, Basu S, Farzal Z, Senior BA. Characterizing nasal delivery in 3D models before and after sinus surgery. Resp Drug Del. 2018. 2018;1:181-188

37. Kimbell JS, Basu S, Garcia GJ, et al. Upper airway reconstruction using long-range optical coherence tomography: Effects of airway curvature on airflow resistance. Lasers Surg Med. 2019:51(2):150-160.

38. Shrestha K, Salati $H$, Fletcher D, Singh $\mathrm{N}$, Inthavong K. Effects of head tilt on squeeze-bottle nasal irrigation-A compu- tational fluid dynamics study. J Biomech. 2021:123:110490

39. Longest PW, Vinchurkar S. Validating CFD predictions of respiratory aerosol deposition: effects of upstream transition and turbulence. J Biomech. 2007:40(2):305-316.

40. Hosseini S, Schuman TA, Walenga R, Wilkins Jr JV, Babiskin A, Golshahi L. Use of anatomically-accurate 3-dimensional nasal airway models of adult human subjects in a novel methodology to identify and evaluate the internal nasal valve. Comp Biol Med. 2020;123:103896.

41. Doorly D, Taylor D, Schroter R. Mechanics of airflow in the human nasal airways. Resp Physiol Neurobiol. 2008;163(1-3):100-110.

42. Baghernezhad N, Abouali O. Different SGS models in large eddy simulation of $90^{\circ}$ square cross-section bends. J Turbulence. 2010(11):N50

43. Saksono P, Nithiarasu P, Sazonov I, Yeo S. Computational flow studies in a subjectspecific human upper airway using a oneequation turbulence model. Influence of the nasal cavity. Int. J. Numer. Meth. Engng 2011;87(1-5):96-114.

44. Nithiarasu P, Hassan O, Morgan K, et al. Steady flow through a realistic human upper airway geometry. Int. J. Numer. Meth. Fluids. 2008;57(5):631-651.

45. Nithiarasu P. Patient-specific modelling of cardiovascular and respiratory flow problems-challenges. In: Computational Biomechanics for Medicine. Springer; 2010:3-3.

46. Nithiarasu P. Biofluid Dynamics (e-book) 2021

47. Lopez-Vidriero MT, Charman J, Keal E, De Silva D, Reid L. Sputum viscosity: correlation with chemical and clinical features in chronic bronchitis. Thorax. 1973;28(4):401408 .

48. Brosseau LM, Roy CJ, Osterholm MT. Facial masking for covid-19. New Eng J Med. 2020;383(21):2092-2093

49. Ryan KA, Bewley KR, Fotheringham SA, et al. Dose-dependent response to infection with SARS-CoV-2 in the ferret model and evidence of protective immunity. Nature Comm. 2021:12(1):1-13.

50. Wang D, Wang Y, Sun W, et al. Population bottlenecks and intra-host evolution during human-to-human transmission of SARSCoV-2. Frontiers in medicine. 2021:8.

51. Watanabe T, Bartrand TA, Weir MH, Omura T, Haas CN. Development of a dose-response model for SARS coronavirus. Risk Analysis. 2010;30(7):1129-1138.

52. Venkatakrishnan $A$, Anand $P$, Lenehan $P$, et al. Antigenic minimalism of SARS-CoV-2 is linked to surges in COVID-19 community transmission and vaccine breakthrough infections. medRxiv. 2021

53. Basu S, Stremler MA. Exploring the dynamics of ' $2 P^{\prime}$ wakes with reflective symmetry using point vortices. J Fluid Mech. 2017;831:72-100.

54. Basu S. Dynamics of vortices in complex wakes: modeling, analysis, and experiments, 
Virginia Polytechnic Institute and State University; 2014.

55. Batchelor CK, Batchelor G. An introduction to fluid dynamics. Cambridge university press; 2000

56. Sato K, Chitose Si, Sato F, Umeno H. Deglutition and respiratory patterns during sleep in the aged with OSAS. Laryngoscope Invest Otolaryngol. 2018;3(6):500-506.

57. Valbuza JS, de Oliveira MM, Zancanella E, et al. Swallowing dysfunction related to obstructive sleep apnea: a nasal fibroscopy pilot study. Sleep Breath. 2011;15(2):209213.

58. Bhutada AM, Broughton WA, Garand KLF Obstructive sleep apnea syndrome (OSAS) and swallowing function-a systematic review. Sleep Breath. 2020:1-9.

59. Sato K, Chitose S-i, Sato K, Sato F, Ono T, Umeno $H$. Recurrent aspiration pneumonia precipitated by obstructive sleep apnea. Auris Nasus Larynx. 2021;48(4):659-665.

60. McSharry D, Malhotra A. Potential influences of obstructive sleep apnea and obesity on COVID-19 severity. J Clin Sleep Med. 2020;16(9):1645-1645

61. Bhatraju PK, Ghassemieh BJ, Nichols M, et al. Covid-19 in critically ill patients in the Seattle region-case series. New Eng J Med. 2020;382(21):2012-2022.

62. Arentz M, Yim E, Klaff L, et al. Characteristics and outcomes of 21 critically ill patients with COVID-19 in Washington State. Jama. 2020;323(16):1612-1614.

63. Tufik S, Gozal D, Ishikura IA, Pires GN, Andersen ML. Does obstructive sleep apnea lead to increased risk of COVID-19 infection and severity? J Clin Sleep Med. 2020;16(8):1425-1426.

64. Cade BE, Dashti HS, Hassan SM, Redline S, Karlson EW. Sleep apnea and COVID-19 mortality and hospitalization. Am J Resp Critical Care Med. 2020;202(10):1462-1464.

65. Maas MB, Kim M, Malkani RG, Abbott SM Zee PC. Obstructive sleep apnea and risk of COVID-19 infection, hospitalization and respiratory failure. Sleep Breath.
2021;25(2):1155-1157

66. Clinic C. Can PAP Therapy Machines Increase the Risk of Spreading the Coronavirus? 2020.

67. Aslam M, Vaezi MF. Dysphagia in the elderly Gastroenterol Hepatol. 2013:9(12):784.

68. Raber-Durlacher JE, Brennan MT, Verdonckde Leeuw IM, et al. Swallowing dysfunction in cancer patients. Supp Care Cancer 2012;20(3):433-443.

69. Suttrup I, Warnecke T. Dysphagia in Parkinson's disease. Dysphagia. 2016;31(1):24-32.

70. Rutten JJ, van Loon AM, van Kooten J, et al. Clinical suspicion of COVID-19 in nursing home residents: symptoms and mortality risk factors. J Am Med Direct Assoc. 2020;21(12):1791-1797. e1791.

71. Vignatelli L, Zenesini C, Belotti LM, et al. Risk of hospitalization and death for COVID-19 in people with Parkinson's disease or parkinsonism. Mov Disord. 2021;36(1):1-10.

72. Kuderer NM, Choueiri TK, Shah DP, et al. Clinical impact of COVID-19 on patients with cancer (CCC19): a cohort study. Lancet. 2020;395(10241):1907-1918.

73. CDC. COVID-19 Hospitalization and Death by Age. 2020

74. Van Egeren D, Novokhodko A, Stoddard $M$, et al. Risk of rapid evolutionary escape from biomedical interventions targeting SARS-CoV-2 spike protein. PloS one. 2021;16(4):e0250780.

75. Van Egeren D, Novokhodko A, Stoddard M et al. Risk of evolutionary escape from neutralizing antibodies targeting SARS-COV-2 spike protein. medRxiv. 2020.

76. Thomson EC, Rosen LE, Shepherd JG, et al. The circulating SARS-CoV-2 spike variant N439K maintains fitness while evading antibody-mediated immunity. BioRxiv. 2020.

77. Starr TN, Greaney AJ, Hilton SK, et al. Deep mutational scanning of SARS-CoV-2 receptor binding domain reveals constraints on folding and ACE2 binding. Cell. 2020;182(5):1295-1310. e1220.

78. Kemp SA, Collier DA, Datir RP, et al. SARS-
CoV-2 evolution during treatment of chronic infection. Nature. 2021;592(7853):277-282.

79. Chen L, Zody MC, Di Germanio C, et al. Emergence of multiple SARS-CoV-2 antibody escape variants in an immunocompromised host undergoing convalescent plasma treatment. Msphere. 2021;6(4):e00480-00421.

80. Van Egeren D, Novokhodko A, Stoddard $\mathrm{M}$, et al. Controlling long-term SARS-CoV-2 infections can slow viral evolution and reduce the risk of treatment failure. Sci Rep. 2021;11(1):1-9.

81. Li G, Li W, He X, Cao Y. Asymptomatic and presymptomatic infectors: hidden sources of coronavirus disease 2019 (COVID-19). Clin Infect Dis. 2020;71(8):2018-2018.

82. Delshad SD, Almario CV, Chey WD, Spiegel BM. Prevalence of gastroesophageal reflux disease and proton pump inhibitor-refractory symptoms. Gastroenterol. 2020;158(5):1250-1261. e1252.

83. Richter JE, Rubenstein JH. Presentation and epidemiology of gastroesophageal reflux disease. Gastroenterol. 2018;154(2):267-276.

84. Lao Y, Joseph-McCarthy D, Chakravarty A, et al. Identifying the optimal parameters for sprayed and inhaled drug particulates for intranasal targeting of SARS-CoV-2 infection sites. arXiv preprint arXiv:201016325. 2020

\section{Saikat Basu}

Department of Mechanical Engine-

ering

South Dakota State University

Brookings

SD

United States

E-mail: Saikat.Basu@sdstate.edu 